\title{
Post Radiotherapy "Isolated LP of the Lips" in a Non-Hodgkin Lymphoma Patient: A Possible Relation
}

\author{
Hamdi H. Shelleh ${ }^{1}$, Husni S. Al Hateeti ${ }^{2}$, Hamad A. Al Fahhad ${ }^{3}$, Sarosh A. Khan ${ }^{4}$, Latif A. Khan ${ }^{2}$, \\ Khalid Bahamdan ${ }^{5}$ \\ ${ }^{1}$ Dermatology Department, Nagran General Hospital, Najran, KSA; ${ }^{2}$ Hematology, MRCP, London, UK; ${ }^{3}$ Dermatology, Najran Uni- \\ versity, Najran, KSA; ${ }^{4}$ Medicine, On-Line Physician Academy, Sirangar, India; ${ }^{5}$ Dermatology, King Khalid University, Abha, KSA. \\ Email: hhs_s2000@hotmail.com
}

Received May 21 ${ }^{\text {st }}, 2012$; revised June $30^{\text {th }}, 2012$; accepted July $12^{\text {th }}, 2012$

\begin{abstract}
We report upon a case of 62 years old Saudi male with non-Hodgkin lymphoma who developed lichen planus (LP) on the inner aspect of the upper lip six months after finishing radiotherapy. The diagnosis of LP was confirmed by histopathology. Literature review reveals few countable similar reports of "post radiotherapyoral LP (OLP)". However, the isolated location of lichen planus on the upper lip per se in this case merits reporting being exceptional and reported before as isolated lichen planus of the lips (ILPL). We assume, after screening literature, that this is the first report of post radiotherapy ILPL in Arabian Gulf countries, though it may be underestimated and underreported. The etiological relation between radiotherapy and LP is discussed.
\end{abstract}

Keywords: LP; OLP; Isolated Lichen Planus of the Lip; ILPL; Lymphoma; Radiotherapy Induced LP

\section{Introduction}

OLP is a chronic T-cell mediated inflammatory disease of the oral mucosa of unknown etiology which rarely undergoes spontaneous remission; rather, it has the potential to become malignant [1]. Isolated LP of the lip is a known condition which was first reported in 1995 [2]. Several reports succeeded, in which the lower lip was the mostly involved site, but in some the upper lip was. This condition may be underestimated and therefore underreported in medical literature. This may explain the scarcity of this entity in literature, and justify reporting it from this region.

\section{Case Report}

A 62 years old Saudi male (Figure 1), attended skin OPD with black ulcerative lesion on the oral aspects of upper lip since 4 months. The lesion was painful while eating especially when ulcerations existed. He was markedly anxious for his lesion as he had had a past history of lymphoma and was scared of a possible linkage between both. He was not on drugs and had no other skin lesions elsewhere. Likewise, he was not a smoker, and denied any tobacco chewing. The history of lymphoma started since March 1999 as a submandibular enlarged lymphnode with general body aches, fever, decreased appetite, and rapid weight loss. The patient was referred to King Faisal Specialist Hospital (KFSH), Riyadh, where he was examined, biopsied and diagnosed as "grade I follicular small cell non Hodgkin lymphoma" according to the final report given to him. Aspirate of bone marrow was positive for malignant cell suggesting the dissemination the disease. This was confirmed by emittion tomography scanning which proved positive in submandibular region, sternum, ribs, lumber spine $\mathrm{V}$ and sacral I. On another visit in April 2001 there was progression of the disease in the mediastinum. On December 2001 he presented with a new painful swelling on the right scapular region

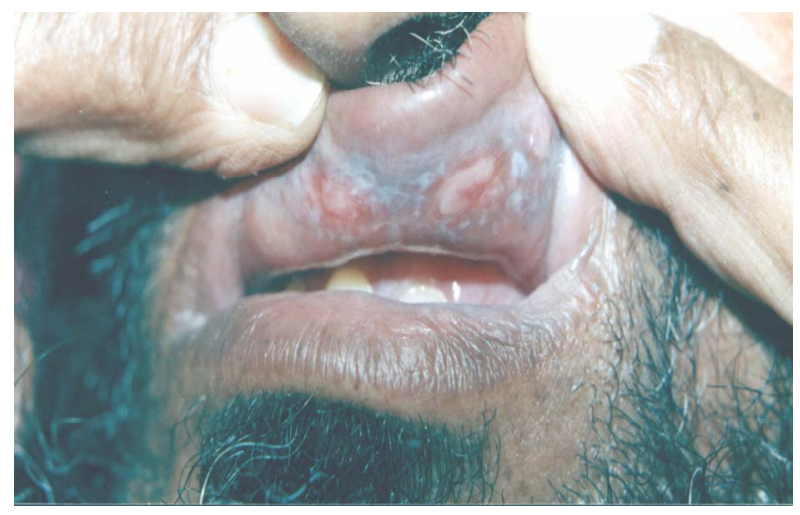

Figure 1. Superficial ulcerations on reticulate hyperpigmented erythematous ill defined surface limited to the inner aspect of upper lip. 
which proved on CT to be a mass in the deltoid muscle with bone changes in the acromial head. There was abdominal lymphadenopathy as well. A new fine needle aspiration revealed AB lymphoid cells and large follicular simple cells. The scenario of treatment was decided to be a combination of radio-chemotherapy for 6 therapy cycles. Accepting that, he went on with the treatment after which he markedly improved. The entire body hair fell down, but restored again after 6 months of therapy. He was on regular follow-up at the oncology centre for 10 months. The current clinical examination at the dermatology clinic revealed diffuse lattice-like whitish lesion on hyperpigmented flat surface occupying the entire inner aspect of the upper lip with few superficial ulcerations. The lesion was not infiltrated; there were no enlarged submandibular lymphnodes. The upper anterior gingiva had a furry appearance with whitish discoloration. The rest of the oral cavity was thoroughly intact and so were the skin and skin appendages elsewhere. The histopathological examination of the lip revealed an epidermis with hyperkeratosis and acanthosis, the upper dermis was occupied with a dense band of lymphocytic infiltrate along the epidermal-dermal junction, suggesting the diagnosis of lichen planus. Atypical cells of any type could not be found. Routine laboratory investigations revealed: WBC: $6540 / \mathrm{mm}$ (seg. 31.7\%, mono. 6.7\%, eosinocytes. $1.7 \%$, and lymphocytes 59.8\%), ESR: $6 \mathrm{~mm} / 1 \mathrm{st} \mathrm{h}$. No other reports of significance. Liver Function Tests were within normal limits, and so were the hepatic viral markers.

\section{Discussion}

Oral lichen planus is a relatively frequent inflammatory mucocutaneous disease of middle-aged patients, affecting approximately $1.27 \%$ of the world population. The association with other immune diseases and the damage to the basal keratinocytes in lichen planus supports an autoimmune aetiology mediated by autocytotoxic T-cells. This case of OLP is of the erosive form. OLP in this case is confined to the upper lip. We think that the special location aforementioned and the special timing; six months after cessation of lymphoma radiotherapy make it worth reporting. The patient was anxious for his oral lesion might be a recurrence of lymphoma, and we were, on our side, anxious too; whether his oral LP could be radiotherapy induced condition. Nevertheless, dermatology is a branch of terminology; let aside the relation of LP with malignancy and radiotherapy which need further studies to settle, this case has special dermatological significance in terms of the location exclusively on the oral mucosa -hence called oral LP in isolation - and the allocation merely on the inner aspect of upper lip-called isolated lichen planus of the lips. Thus, it is sub type of sub-type of LP. However, we do not get engaged a lot in such di- visions and subdivisions till proved significant, as LP at last is LP, apart from such distribution and localization. Oral involvement occurs as part of LP disease in 50\% of LP patient population, even $60 \%-70 \%$ in another study [3]. It may precede, accompany or follow lesions elsewhere on the skin in the context of LP disease. "OLP in isolation" occurs in $20 \%-30 \%$ of the patient population [4], it affects $1 \%-2 \%$ of population in general and has the same histopathology of cutaneous LP. It may develop on any site of the oral mucosa and usually asymptomatic except for the atrophic and erosive patterns which may cause burning sensation and trouble eating. For easier clinical approach, OLP may take diverse patterns or presentations which include [3,5]: 1) The reticular pattern: it is the most common pattern of OLP, it is presented as reticulated white papular streaks; 2) The papular pattern: usually seen together mixing within the reticulate pattern; 3) The plaque pattern: resembles leukoplakia, the border of the plaque is reticulate as in type 1 ; 4) The atrophic pattern: also the border is reticulate here; 5) The bullous LP is rarely encountered; 6) The desquamative gingivitis; 7) The gingival stomatitis; 8) Erosive/ulcerative LP: this predominates in the elderly, but it is comparatively uncommon pattern. Ulcers develop secondary to atrophic lesion or blisters, they are irregular shaped with slough, but the red halo and whitish striae are pathognomonic of oral LP. Our patient belongs to this type of clinical presentation.

Though any of these types may occur alone, the clinical picture may be a mixture of more than one. Location wise, the buccal "gingival and glossal" mucosae are the most commonly affected sites. But the palate, floor of month, lips and any area of the oral cavity may also be affected. LP of the lips usually has oral lesions elsewhere, but when in isolation they are reported as ILPL [6]. A bipolar LP of gingiva and vulva has been described as vulvovaginal-gingival LP syndrome [7] in which other mucosae may be involved as well. In $1 \%$ of patient population, esophagus is the only site involved with LP [8], this represents a singular mucosal involvement of OLP as on the lip, and similar to the singular involvement of the glans or vulva. Again it is not known why a small particular area of mucosa or skin only reacts with a disease which is supposed to be of a systemic immunological mechanism in the absence of localizing factors like radiotherapy or other irritating and mediating factors. For instance, OLP may occur adjacent to gold, mercury and other amalgam dental fillings. This assumes a role of these elements in the pathogenesis of localized LP. However, our patient has not been using any of the suspected drugs, and had no close adjuvant dental fillings to be incriminated, whereas, radiotherapy was the only preceding condition to presume a potential correlation. Considerable evidence now exist that the etiological process 
underlying LP is auto-immune mediated. Langerhans cells increase in the early lesion of LP, and the dermal infiltrate consists largely of T-cells, $40 \%$ of these T-cells in the dermal epidermal junction and $80 \%$ in the dermis are of helper/inducer subset (CD4+). The T-cells represent an antigen-stimulated cell. This antigen, whether it be a virus or drug carried on the cell surface, could alter the T-cell interpretation of the histocompatibility complex so that it recognizes it as foreign and attacks it. A constant feature of the lymphocytes in lichenoid reactions is their epidermotropism; the affinity for the epidermis. Adjacent keratinocytes produce a series of cytokines that play a critical role in attracting lymphocytes within the epidermis. The keratinocyte-lymphocyte interaction is important in the pathogenesis of Lichenoid reactions. In the primary phase of OLP IL-12 may drive the pathological destruction in OLP lesions by elevating IFN-gamma protein locally. IFN-gamma may play an important role for the pathological destruction in OLP lesions [9], but in monitoring the disease OLP activity serum IL-8 level was found more sensitive marker than serum IL-6 [10]. However, serum and salivary IL-18 are elevated as well correlating with the severity of illness. This may improve the predictive or prognostic values of inflammatory cytokines for OLP [11]. Though it is an idiopathic disease a relation to a chronic liver disease or immunodeficiency has been proposed. The association of erosive LP with primary biliary cirrhosis and chronic active hepatitismay be related to an associated immunological pathogenesis [12], or else, to an iatrogenic cause, as some patients have received penicillamine therapy which was reported as a triggering factor of LP. Hepatitis $\mathrm{C}$ virus infection was reported in significant ratio of LP as well [13]. This puts a task on oral physicians and dentists to pay special attention in any OLP lesion, and perform laboratory screening to rule out these possible associations. An increased incidence of glucose tolerance test in LP has been reported. Lichen planus may be induced by neoplasia; paraneoplastic LP, and a cell-mediated immune reaction possibly backs this association. It was reported with thymoma, autoimmune diseases and Castleman's tumor [14-16]. After tumor resection LP lesions regressed and melanosed. It is recommend that with patients presenting a severe, therapy- resistant, erosive stomatitis, one should be alert to the possibility of underlying immunological diseases and/or tumors [17]. OLP per se carries premalignant potential, and a significant ratio of it may transform to malignancy over years $[1,18]$. Similar to this case, Sporadic cases of generalized or localized post radiotherapy LP have been reported, being treated for different types of cancers, but little is known about the mechanism. [19-24]. We assume that LP-radiotherapy relation in these patient is not fortuitous, OLP has developed as a result of radiological trauma, besides the activation of $\mathrm{T}$ lympho- cytes during carcinogenesis and/or radiation thru an autoimmune mechanism. The isomorphic; or the "isoradiotopic" response of skin to radiological trauma (Koebner's phenomenon) may issue as mucositis - the most common complications of radiotherapy $[20,23,24]$. Mucositis mediates the pathogenic process and forms an inflammatory basis for OLP. In addition to this, lymphocytes of CD8+ and CD4+ type become over sensitized to tumor antigens of lymphoma cells, and/or get altered by frequent exposure to radiotherapy, a condition of dysimmuno-reactivity or immuno-discrepancy ensues which bring about the intrinsic production of various autoantibodies. These attack the cross-reacting antigenic structures in the skin and/ or mucosae in an autoimmune process to produce the clinical picture of LP or OLP causing cellular degeneration, apoptosis and disruption of the basal membrane. But why on the upper lip in this patient? The upper lip was the most neighbouring region that was directly and mostly hit by radiation therapy causing a mucosal injury, and indirectly, by the mucosal damage resulting via an autoimmune mechanism. Hence, we think that lichen planus has windows, directly opened with both neoplasms and radiotherapy here allowing the interplay of traumatic, humoral and cell-mediated responses in LP pathogenesis [25]. We found, after screening literature, that this is the first case report of OLP induced by radiotherapy in Saudi Arabia and in the Arabian Gulf countries. As in cutaneous LP, there is no specific treatment for OLP. New oral retinoids (Temarotene, Feurelinide) appear promising. Topical cyclosporine has equivocal value. New trials support a significant response of OLP to the topical immunemodulator; tacrolimus [26]. Levamisole increases cellular immunity in many autoimmune and inflammatory diseases. There has been significant success with Levamisolemonotherapy, and dramatic response to levamisole with low dose prednisolone [26,27]. However, regular follow-up, including biopsy, is important especially in resistant erosive OLP in order to early pick up any suspected malignant transformation. Once our patient was reassured, he was missed on follow up, and appeared again, 5 years later; healthy, but still with OPL, still limited to the upper lip, and still without signs of transformation, or recurrence of lymphoma. It was a survive!

\section{Conclusion}

Thus, radiotherapy elicited OLP may becomea true entity, that needs further studies for confirmation. It may be due to 2 factors: 1) traumatic radiological mucositis which may act as a Koebner's sign; 2) and/or autoimmune mechanism resulting from the aberrant cancer cells and/or radiotherapy mutilating effect. OLP may be of the ILPL type if lips were more adjuvant to irradiation source. This is the first case of ILPL reported from KSA and Arabian 


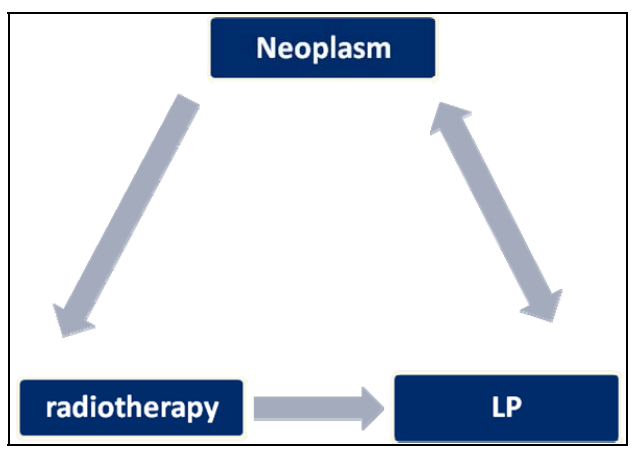

Diagram 1. Cancer-radiotherapy-lichen planus-triangle; the key ward is possibly the T-lymphocyte which gets disrupted in-between the sharp angles.

Gulf countries, but it may be underestimated and under reported. Doctors should be aware that neoplasms and/or radiotherapy for neoplasms may induce OLP and OLP may transform, this mandates awareness by the radiologist to focus their radiation beam as far as possible far from the mucosae; it is fragile and may get easily trapped in LP. Further research is required to elucidate the interrelations in the cancer-radiotherapy-LP triangle; the key ward is possibly the T lymphocyte which gets disrupted in between the sharp angles (Diagram 1).

\section{REFERENCES}

[1] R. A. Safadi, S. Z. Al Jaber, H. M. Hammad and A. A. Hamasha, "Oral Lichen Planus Shows Higher Expressions of Tumor Suppressor Gene Products of p53 and p21 Compared to Oral Mucositis. An Immunohistochemical Study," Archives of Oral Biology, Vol. 55, No. 6, 2010, pp. 454-461. doi:10.1016/j.archoralbio.2010.03.019

[2] P. H. Itin, P. Schiller, L. Gilli and S. A. Buechner, "Isolated Lichen Planus of the Lip," British Journal of Dermatology, Vol. 132, No. 6, 1995, pp. 1000-1002. doi:10.1111/j.1365-2133.1995.tb16963.x

[3] D. Eisen, "The Clinical Features, Malignant Potential, and Systemic Associations of Oral Lichen Planus: A Study of 723 Patients," Journal of the American Academy of Dermatology, Vol. 46, No. 2, 2002, pp. 207-214. doi: $10.1067 / \mathrm{mjd} .2002 .120452$

[4] F. Lozada-Nur and C. Miranda, "Oral Lichen Planus: Epidemiology Clinical Characteristics, and Associated Diseases," Seminars in Cutaneous Medicine and Surgery, Vol. 16, No. 4, 1997, pp. 273-277. doi:10.1016/S1085-5629(97)80016-8

[5] S. Silverman and S. Bahl, "Oral Lichen Planus Update: Clinical Characteristics, Treatment Responses, and Malignant Transformation," American Journal of Dentistry, Vol. 10, No. 6, 1997, pp. 259-263.

[6] S. J. R. Alan and P. K. Buxon, "Isolated Lichen Planus of the Lip," British Journal of Dermatology, Vol. 135, No. 1, 1996, pp. 145-146.

doi:10.1111/j.1365-2133.1996.tb03631.x

[7] D. Eisen, "The Vulvovaginal-Gengival Syndrome of Li- chen Planus, the Clinical Characteristics of 22 Cases," Archives of Dermatology, Vol. 130, No. 11, 1994, pp. 13791382. doi:10.1001/archderm.1994.01690110045004

[8] L. P. Fox, C. J. Lightdale and M. E. Grossman, "Lichen Planus of the Esophagus: What Dermatologists Need to Know," Journal of the American Academy of Dermatology, Vol. 65, No. 1, 2011, pp. 175-183. doi:10.1016/i.jaad.2010.03.029.

[9] Y. X. Pan, Y. Cai and H. B. Yu, "Expression of Interleukin-12p40 and Interferon-Gamma in Local Lesions of Human Oral Lichen Planus," West China Journal of Stomatology, Vol. 29. No. 2, 2011, pp. 179-182.

[10] A. Sun, J. T. Wang, J. S. Chia and C. P. Chiang, "Serum Interleukin-8 Level Is a More Sensitive Marker than Serum Interleukin-6 Level in Monitoring the Disease Activity of Oral Lichen Planus," British Journal of Dermatology, Vol. 152, 2005, pp. 1187-1192. doi:10.1111/j.1365-2133.2005.06497.x

[11] Y. Zhang, W. Liu, S. Zhang, H. Dan, R. Lu, F. Wang, J. Wang, M. Zhou, X. Zeng, M. Lin, Q. Chen and Y. Zhou, "Salivary and Serum Interleukin-18 in Patients with Oral Lichen Planus: A Study in an Ethnic Chinese Population," Inflammation, Vol. 35, No. 2, 2012, pp. 399-404.

[12] M. Carbone, P. G. Arduino, M. Carrozzo, S. Gandolfo, M. R. Argiolas, G. Bertolusso, D. Conrotto, M. Pentenero and R. Broccoletti, "Course of Oral Lichen Planus: A Retrospective Study of 808 Northern Italian Patients," Oral Diseases, Vol. 15, No. 3, 2009, pp. 235-243. doi:10.1111/j.1601-0825.2009.01516.x

[13] S. Petti, M. Rabiei, M. De Luca and C. Scully, "The Magnitude of the Association between Hepatitis C Virus Infection and Oral Lichen Planus: Meta-Analysis and Case Control Study," Odontology, Vol. 99, No. 2, 2011, pp. 168178. doi:10.1007/s10266-011-0008-3

[14] J. Qiao, G. Zhou, Y. Ding, D. Zhu and H. Fang, "Multiple Paraneoplastic Syndromes: Myasthenia Gravis, Vitiligo, Alopecia Areata, and Oral Lichen Planus Associated with Thymoma," Journal of the Neurological Sciences, Vol. 308, No. 1, 2011, pp. 177-179. doi:10.1016/j.jns.2011.05.038

[15] Y. Kaku, N. Shimamoto, H. Matsunaga, M. Makiura, A. Fujisawa and K. Morita, "Oral Erosive Lichen Planus and Alopecia Areata with Good's Syndrome (Thymoma with Hypogammaglobulinemia)," European Journal of Dermatology, Vol. 21, No. 1, 2011, pp. 124-125.

[16] V. Mahler, C. Antoni, G. J. Anhalt, H. J. Koch, K. P. Peters, B. Manger, et al., "Graft-versus-Host-Like Mucocutaneous Eruptions with Serological Features of Paraneoplastic Pemphigus and Systemic Lupus Erythematosus in a Patient with Non-Hodgkin's Lymphoma," Dermatology, Vol. 197, No. 1, 1998, pp. 78-83. doi:10.1159/000017964

[17] C. J. Hsiao, M. M. Hsu, J. Y. Lee, W. C. Chen and W. C. Hsieh, "Paraneoplastic Pemphigus in Association with a Retroperitoneal Castleman's Disease Presenting with a Lichen Planuspemphigoides-Like Eruption. A Case Report and Review of Literature," British Journal of Dermatology, Vol. 144, No. 2, 2001, pp. 372-376. doi:10.1046/j.1365-2133.2001.04030.x

[18] S. Poomsawat, W. Buajeeb, S. O. Khovidhunkit and J. 
Punyasingh, "Overexpression of cdk4 and p16 in Oral Lichen Planus Supports the Concept of Premalignancy," Journal of Oral Pathology \& Medicine, Vol. 40, No. 4, 2011, pp. 294-299.

doi:10.1111/j.1600-0714.2010.01001.x

[19] N. Morar and N. D. Francis, "Generalized Lichen Planus Induced by Radiotherapy: Shared Molecular Mechanisms?" Clinical and Experimental Dermatology, Vol. 34, No. 7, 2009, pp. e434-e435. doi:10.1111/j.1365-2230.2009.03434.x

[20] M. Pretel and A. España, "Lichenplanus Induced by Radiotherapy," Clinical and Experimental Dermatology, Vol. 32, No. 5, 2007, pp. 582-583. doi:10.1111/j.1365-2230.2007.02449.x

[21] M. Eichbaum, W. Harms, S. Bolz, A. Schneeweiss and C. Sohn, "Generalized Lichen Ruberplanus - Induced by Radiotherapy of the Breast?" Onkologie, Vol. 29, No. 11, 2006, pp. 521-523. doi:10.1159/000096048

[22] J. H. Kim and S. J. Krivda, "Lichen Planus Confined to a Radiation Therapy Site," Journal of the American Academy of Dermatology, Vol. 46, No. 4, 2002, pp. 604-605. doi: $10.1067 / \mathrm{mjd} .2002 .119654$
[23] D. Shurman, H. L. Reich and W. D. James, "Lichen Planus Confined to a Radiation Field: The 'Isoradiotopic' Response," Journal of the American Academy of Dermatology, Vol. 50, No. 3, 2004, pp. 482-483. doi:10.1016/S0190-9622(03)02144-3

[24] G. F. Sciallis, C. L. Loprinzi and M. D. Davis, "Lichen Planus Induced by Radiotherapy," British Journal of Dermatology, Vol. 152, 2005, pp. 399-401.

[25] Y. N. Wang, H. C. Chen, B. Wang and H. Fang, "Oral and Esophageal Lichen Planus Following Radiotherapy of Nasopharyngeal Carcinoma," Acta Dermato-Venereologica, Vol. 91, No. 1, 2011, pp. 94-95.

[26] J. A. Byrd, M. D. Davis, A. J. Bruce, L. A. Drage and R. S. Rogers, "Response of Oral Lichen Planus to Topical Tacrolimus in 37 Patients," Archives of Dermatology, Vol. 140, No. 12, 2004, pp. 1508-1512. doi:10.1001/archderm.140.12.1508

[27] T. H. Won, S. Y. Park, B. S. Kim, P. S. Seo and S. D. Park, "Levamisole Monotherapy for Oral Lichen Planus," Annals of Dermatology, Vol. 21, No. 3, 2009, pp. 250254. doi:10.5021/ad.2009.21.3.250 